\title{
Leukocyte neutrophil ratio (NLR) as biomarker for neuroinflammation in patients with intracerebral haemorrhage and traumatic brain injury
}

\author{
Amrita Ghosh ${ }^{1}$, Ranabir Pal' ${ }^{2}$, Rakesh Mishra ${ }^{3}$, Rafael Cincu ${ }^{4}$, \\ Luis Rafael Moscote-Salazar ${ }^{5}$, Amit Agrawal ${ }^{3}$ \\ ${ }^{1}$ Department of Biochemistry, Calcutta Medical College, Kolkata, India \\ ${ }^{2}$ Department of Community Medicine, MGM Medical College and Hospital, Bihar, India \\ ${ }^{3}$ Department of Neurosurgery, All India Institute of Medical Sciences, Bhopal, Madhya Pradesh, India \\ ${ }^{4}$ Department of Neurosurgery, General University Hospital, Valencia, Spain \\ ${ }^{5}$ Department of Neurosurgery, University of Cartagena, Cartagena de Indias, Colombia
}

Circulating inflammatory markers may predict the broad clinical spectrum of traumatic brain injury (TBI). The present study aimed to illustrate the role of leukocytes, precisely neutrophil and lymphocytes, in neuroinflammation as a new vista in neuro-critical care. Search terms were: elevations in peripheral blood leukocytes counts, neutrophil, lymphocyte with precisely neutrophil to lymphocyte ratios, associated with adverse outcomes of traumatic brain injury, pre and post-operative conditions of intracerebral haemorrhage, seizures, delirium, non-convulsive status epilepticus, confusion, aphasia, agitation, coma, disability and death. As a result, we identified 16 publications describing leukocyte biomarkers associated with neuroinflammation following TBI from PubMed, Cochrane Registry of Controlled Trials, Medline and Embase (Ovid) on randomised controlled trials (RCTs), non-RCTs and cohort studies published in the last decade. This study concluded that elevations in peripheral blood leukocyte and neutrophil to lymphocyte ratios could predict adverse outcomes of a cerebral haemorrhage.

Keywords: mirror therapy (MT), motor imagery training, lower extremity (LE), randomized controlled trial $(\mathrm{RCT})$, rehabilitation, motor recovery, spasticity, functional ability, balance

\section{INTRODUCTION}

Leukocyte as neuroinflammation biomarkers has been centre of research interest for the clinicians of the neuroscience speciality in the new millennium (1-3). Neutrophil to lymphocyte ratio (NLR) calculated by dividing the absolute neutrophil count (ANC) by the absolute lymphocyte count (ALC) is a biomarker of a systematic inflammatory response (4). Several research groups have extensively studied the roles of different inflammatory cells in TBI viz. leukocytes with particular attention to neutrophil and lymphocyte and their interrellation $(5,6)$. NLR has been shown to be reliably related to the unfavourable outcomes in intracerebral haemorrhage (ICH) $(7,8)$, predictor of outcome in critical illness including patients with severe TBI (sTBI) (9). Research groups found NLR to be a predictive factor in different diseases, including cardiovascular problems, cancer $(10,11)$ and other neurological conditions viz. stroke, mild TBI (mTBI), and pediatric brain tumours (7,12-16). Leukocytosis, even without associated infection or fever, correlates with the extent of brain damage in the immediate post-operative period following major intracranial surgeries with values as high as $50,000 / \mathrm{mm}^{3}$ WBC count in the post-operative period following hemi- 
spherectomy for intractable epilepsy (17). In pediatric TBI, prolonged immune response during secondary brain injury correlated with the changes in neutrophils, lymphocytes and NLR, though there was a lack of correlation between NLR and GOS-E at admission (18). The current study aimed to determine whether elevations in peripheral blood leukocytes counts, precisely neutrophil to lymphocyte ratio, were associated with adverse outcomes in intracerebral haemorrhage and traumatic brain injury (TBI).

\section{PATHOPHYSIOLOGY FOLLOWING NEUROTRAUMA}

The neuro-inflammatory cascade after traumatic brain injury (TBI) peaks between 24-48 hours. These are the body responses to clear the devitalised tissue and extravasated blood called inflammation in common parlance (4). Physiologically controlled upregulation of inflammatory mediators provide neuroprotection and complex neuroinflammation after injury. Leukocytes congregate at the damaged areas to reversion the cell damage, eliminate necrotic cells, and support tissue repair (4). Disproportionate upregulation of this inflammatory cascade in the brain or spinal cord causes further damage beyond the initial assault, accelerates neurodegenerative processes, and can lead to permanent neurological impairment by secondary brain damage. The cellular and molecular mediators of this neuro-inflammatory process sneak out into the systemic circulation and, when overwhelmed, lead to systemic responses. Thus these peripheral blood inflammatory circulating markers have the potential to predict clinical outcomes in brain damage. Chen et al. (9) analysed NLRs in 855 patients of sTBI for up to one year or death (whichever was earlier) and reported that high NLRs in adult severe TBI cases were associated with unfavourable functional outcomes and mortality. Petrone et al. (14) observed that NLR in clinically mTBI/ concussion may be comparable to sTBI, which signifies that even significant neuronal damage may still not be evident clinically in the short term and hence NLRs should be a part of indicators for triaging, deciding further course of management and long-term follow-up. Zhao et al. (19) proposed a predictive model on 6-month outcome in adult TBI from conventional prognostic factors, including NLR, based on retrospective analysis of 1291 cases of TBI. All these and other studies unanimously point towards the fact that several functions of the neutrophils noted in injury response are for the orchestration of pro-repair mechanisms with connected innate and adaptive immune systems, even though precise roles of neutrophil after post-TBI is yet to be understood (20). The primary role of neutrophils is to get quickly activated post-injury, migrate circulation into the brain injury site. Neutrophils are crucial in the innate immune system, yet inapt or undue activation can cause tissue damage.(14) NLR is related to the hyperacute inflammatory response to cerebral injury, and increased NLR values indicate potential secondary brain injury and susceptibility to the post-stroke extra-cranial complications $(13,21)$.

\section{LEUKOCYTOSIS AND THE NEUROINFLAMMATION}

The common finding across all the studies that high NLR is associated with poorer outcomes points towards the correlation of NLR with initial phases of the pathophysiology of TBI. Consequently, it holds no value just for prognostication but also for optimum management of TBI. Furthermore, this potential may be comparable to subjective parameters viz. GCS, PTA, and LOC in younger populations. NLR is an easily obtained clinical marker that may assist in predicting TBI prognosis and potentially become a target for treatment if pharmacological regulation of neuroinflammation improves TBI outcomes (18). A critical finding in this regard is the relationship of onset of significant lymphocytopenia after polytrauma with poor outcome, and therefore serial white blood cell counts should be done to reflect stressful events in critically ill patients (9). The research group at the University of West Virginia went a step ahead to study changes in gene expression modulating the immune system in addition to NLR in TBI patients. They concluded the relative patterns rather than gene expressions are better predictors of outcomes, and further standardisations are needed to make these diagnostics reliable enough to tailor treatment approaches (14).

\section{NLR AND ICH}

As NLR is a quantitative measure of the balance between innate and adaptive immune responses, an increased NLR may reflect either an increase in neurotrophils or a decrease in lymphocytes or both (14). NLR is emerging as an easy and reliable predictor in various neurological conditions, including $\mathrm{ICH}$. There is increasing evidence to suggest that elevated NLR is associated with poor outcome in patients with ICH $(8,13,22,23)$. Researchers worldwide have noted that inflammation plays a critical role in ICH progression. NLR was identified as a predictor for the short-term outcome in ICH patients, whereas the association with the long-term outcome remains unpredictable. A study assessing the relationship between NLR and the long-term prognosis in ICH patients found that NLR independently predicted 180-day morbidity and 180-day mortality with spontaneous ICH to assess (24). Considering the pos- 
sible influence of surgical evacuation on NLR, a recent retrospective study to investigate the association of NLR and 90-day functional outcome in 104 patients who underwent surgical treatment suggests that NLR at admission could predict the functional outcome of patients with ICH after surgical treatment (25).

\section{PEDIATRIC TBI AND LEUKOCYTES}

A retrospective from the Florida Atlantic University College of Medicine found that reasonably elevated NLRs at 24 and $48 \mathrm{~h}$ post-TBI were associated with worse outcomes in 188 pediatric TBI patients. These findings suggest the utility of NLR as a valuable and cost-effective predictor of outcome in pediatric TBI but need further validation by prospective trials $(4,26)$. Here, it is essential to realise that all the existing prognosticating clinical measures such as the GCS, PTA and LOC are subjective and more so in pediatric patients. Hence a more objective panel like the NLR, which is easy to obtain, may prove to be a better predictive indicator to guide further management. Interestingly this study found a poor correlation between GCS and NLR which prompts for the need to evaluate combined parameters for better prognostication.

\section{NLR IN THE DIAGNOSIS AND PROGNOSIS OF TBI}

Recently, a Chinese research group evaluated the post-operative neutrophil to lymphocyte ratio (NLR) as the prognostic marker for patients with intracerebral haemorrhage (ICH) undergoing surgical hematoma evacuation. They found that admission Glasgow Coma Scale score, initial hematoma volume and post-operative NLR were independently associated with 30-day mortality (5). An Italian study evaluated the relationship between the total and differential leukocyte counts and the NLR at admission with neurological deterioration within seven days post-ICH. They noted that high neutrophil and low lymphocyte counts on admission independently predicted adverse outcomes during the initial week after ICH, and NLR represented a reliable predictive biomarker (8). A TBI consists of two events viz. a. the primary injury, or the original force that causes damage to the neural tissue, $b$. the secondary injury, the body's inflammatory response to the injury that can cause blood-brain barrier disruption, neurotoxicity, neuronal death, and neurodegeneration. As with any injury, TBI causes a neutrophil injury-response, which homeostatically initiates repair processes via interactions with brain resident microglia and peripheral monocyte-derived macrophages (20,27-29).

\section{CONCLUSIONS}

The studies included varied in terms of the study population, study design, tools of measurement and analyses and therefore, the results in this narrative review summarises the literature available but could not establish the level of evidence. The utility of NLR should be explored as a neuroinflammation marker in various neurological disorders in well-controlled study designs. Animal models on neurological disorders and NLR with translational research can identify the link amenable to pharmacological therapy. Though this is not intended to be a systematic review, we conducted our search and identification of articles in a systematic process to minimise the error and include the maximum available literature for this review. Another major strength of the iterative process of searching through multiple electronic databases is to review the most updated overview. We have included studies on traumatic brain injury and intracerebral haemorrhage to indicate the two most common neurosurgical emergencies and leading pathologies resulting in morbidity and mortality worldwide. Our review contains the citations of both adult and pediatric patients; it provides a comprehensive overview across age groups on the utility of NLR.

Conflict of interest: none declared Financial support: none declared

\section{REFERENCES}

1. Simats A, Garcia-Berrocoso T, Montaner J. Neuroinflammatory biomarkers: From stroke diagnosis and prognosis to therapy. Biochim Biophys Acta. 2016;1862(3):411-24.

2. Mehta SL, Manhas N, Raghubir R. Molecular targets in cerebral ischemia for developing novel therapeutics. Brain Res Rev. 2007;54(1):34-66

3. Liesz A, Dalpke A, Mracsko E, Antoine DJ, Roth S, Zhou W, et al. DAMP signaling is a key pathway inducing immune modulation after brain injury. J Neurosci. 2015;35(2):583-98.

4. Zahorec R. Ratio of neutrophil to lymphocyte counts - rapid and simple parameter of systemic inflammation and stress in critically ill. Bratislavske lekarske listy. 2001;102(1):5-14.
5. Albertsmeier M, Quaiser D, von Dossow-Hanfstingl V, Winter H, Faist E, Angele MK. Major surgical trauma differentially affects T-cells and APC. Innate Immunity. 2015;21(1):55-64.

6. Lumsdaine W, Easton RM, Lott NJ, White A, Malmanche TL, Lemmert $K$, et al. Neutrophil oxidative burst capacity for peri-operative immune monitoring in trauma patients. Injury. 2014;45(8):1144-8.

7. Giede-Jeppe A, Bobinger T, Gerner ST, Sembill JA, Sprügel MI, Beuscher VD, Lücking $\mathrm{H}$, Hoelter P, Kuramatsu JB, Huttner HB. Neutrophil-to-Lymphocyte Ratio Is an Independent Predictor for In-Hospital Mortality in Spontaneous Intracerebral Hemorrhage. Cerebrovasc Dis. 2017;44(1-2):26-34. 
8. Lattanzi S, Cagnetti C, Provinciali L, Silvestrini M. Neutrophil-tolymphocyte ratio and neurological deterioration following acute cerebral hemorrhage. Oncotarget. 2017;8(34):57489-94.

9. Chen W, Yang J, Li B, Peng G, Li T, Li L, Wang S. Neutrophil to Lymphocyte Ratio as a Novel Predictor of Outcome in Patients With Severe Traumatic Brain Injury. J Head Trauma Rehabil. 2018 Jan/ Feb;33(1):E53-E59.

10. Chen J, Qu X, Li Z, Zhang D, Hou L. Peak Neutrophil-to-Lymphocyte Ratio Correlates with Clinical Outcomes in Patients with Severe Traumatic Brain Injury. Neurocrit Care. 2019 Apr;30(2):334-339.

11. Chen W, Wang X, Liu F, Tian Y, Chen J, Li G, et al. The Predictive Role of Postoperative Neutrophil to Lymphocyte Ratio for 30-Day Mortality After Intracerebral Hematoma Evacuation. World Neurosurg. 2020;134:e631-e5.

12. Guo R, Wu Y, Chen R, Yu Z, You C, Ma L, et al. Clinical Value of Neutrophil-to-Lymphocyte Ratio in Primary Intraventricular Hemorrhage. World Neurosurg. 2019;127:e1051-e6.

13. Lattanzi S, Cagnetti C, Provinciali L, Silvestrini M. Neutrophil-toLymphocyte Ratio Predicts the Outcome of Acute Intracerebral Hemorrhage. Stroke. 2016;47(6):1654-7.

14. Petrone AB, Gionis V, Giersch R, Barr TL. Immune biomarkers for the diagnosis of mild traumatic brain injury. NeuroRehabilitation. 2017;40(4):501-508.

15. Siwicka-Gieroba D, Malodobry K, Biernawska J, Robba C, Bohatyrewicz R, Rola R, Dabrowski W. The Neutrophil/Lymphocyte Count Ratio Predicts Mortality in Severe Traumatic Brain Injury Patients. J Clin Med. 2019 Sep 12;8(9):1453.

16. Wilson JRF, Saeed F, Tyagi AK, Goodden JR, Sivakumar G, Crimmins D, Elliott M, Picton S, Chumas PD. Pre-operative neutrophil count and neutrophil-lymphocyte count ratio (NLCR) in predicting the histological grade of paediatric brain tumours: a preliminary study. Acta Neurochir (Wien). 2018 Apr;160(4):793-800.

17. Agrawal $D$, Kurwale N, Sharma BS. Leukocytosis after routine cranial surgery: A potential marker for brain damage in intracranial surgery. Asian J Neurosurg. 2016 Apr-Jun;11(2):109-13.

18. Kimball R, Shachar E, Eyerly-Webb S, Patel DM, Spader H. Using the neutrophil-to-lymphocyte ratio to predict outcomes in pediatric patients with traumatic brain injury. Clin Neurol Neurosurg. 2020 Jun;193:105772.
19. Zhao JL, Du ZY, Yuan $Q$, Yu J, Sun YR, Wu X, Li ZQ, Wu XH, Hu J. Prognostic Value of Neutrophil-to-Lymphocyte Ratio in Predicting the 6-Month Outcome of Patients with Traumatic Brain Injury: A Retrospective Study. World Neurosurg. 2019 Jan 3:S18788750(18)32930-9.

20. Liu YW, Li S, Dai SS. Neutrophils in traumatic brain injury (TBI): friend or foe? J Neuroinflammation. 2018 May 17;15(1):146.

21. Dirnagl U, Klehmet J, Braun JS, Harms H, Meisel C, Ziemssen T, et al. Stroke-induced immunodepression: experimental evidence and clinical relevance. Stroke. 2007;38(2 Suppl):770-3.

22. Qin J, Li Z, Gong G, Li H, Chen L, Song B, Liu X, Shi C, Yang J, Yang T, Xu $Y$. Early increased neutrophil-to-lymphocyte ratio is associated with poor 3-month outcomes in spontaneous intracerebral hemorrhage. PLoS One. 2019 Feb 7;14(2):e0211833.

23. Wang F, Xu F, Quan Y, Wang L, Xia JJ, et al. Early increase of neutrophilto-lymphocyte ratio predicts 30 -day mortality in patients with spontaneous intracerebral hemorrhage. CNS Neurosci Ther. 2019 Jan;25(1):30-35.

24. Zhang F, Ren Y, Fu W, Yang Z, Wen D, Hu X, et al. Predictive Accuracy of Neutrophil-to-Lymphocyte Ratio on Long-Term Outcome in Patients with Spontaneous Intracerebral Hemorrhage. World Neurosurg. 2019;125:e651-e7.

25. Zhang F, Tao C, Hu X, Qian J, Li X, You C, Jiang Y, Yang M. Association of Neutrophil to Lymphocyte Ratio on 90-Day Functional Outcome in Patients with Intracerebral Hemorrhage Undergoing Surgical Treatment. World Neurosurg. 2018 Nov;119:e956-e961.

26. Pagowska-Klimek I, Lewkowicz P, Banasik M, Krajewski W, Tchórzewski $\mathrm{H}$. Isolated head injury in children affects the neutrophil function and lymphocyte count. J Trauma. 2007 Jul;63(1):179-86.

27. Corps KN, Roth TL, McGavern DB. Inflammation and neuroprotection in traumatic brain injury. JAMA Neurology. 2015;72(3):355-62.

28. Jassam YN, Izzy S, Whalen M, McGavern DB, El Khoury J. Neuroimmunology of Traumatic Brain Injury: Time for a Paradigm Shift. Neuron. 2017;95(6):1246-65.

29. McKee CA, Lukens JR. Emerging Roles for the Immune System in Traumatic Brain Injury. Front Immunol. 2016 Dec 5;7:556. 